\title{
A New Silver Lining on the Horizon is the Use of Chimeric Antigen Receptors as Therapeutic Option for Autoimmune Hepatitis
}

ISSN: 2637-7632

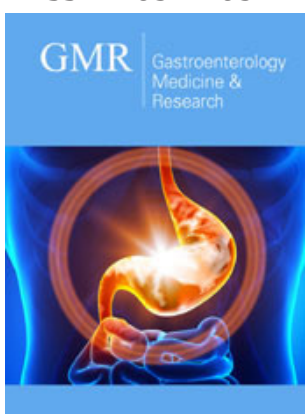

${ }^{* 1}$ Corresponding author: Matthias Hardtke-Wolenski, Department of Gastroenterology and Hepatology, Germany

Submission: April 16, 2019

Published: 泚 May 13, 2019

Volume 3 - Issue 1

How to cite this article: Julia Pietrek, Matthias Hardtke-Wolenski. A New Silver Lining on the Horizon is the Use of Chimeric Antigen Receptors as Therapeutic Option for Autoimmune Hepatitis. A Review of the Literature. Gastro Med Res. 3(1). GMR.000555 2019.

DOI: $10.31031 /$ GMR.2019.03.000555

Copyright@ Matthias Hardtke-Wolenski, This article is distributed under the terms of the Creative Commons Attribution 4.0 International License, which permits unrestricted use and redistribution provided that the original author and source are credited.

\author{
Julia Pietrek and Matthias Hardtke-Wolenski*
}

Department of Gastroenterology and Hepatology, Germany

\begin{abstract}
Autoimmune hepatitis (AIH) is a severe autoimmune inflammation that needs life-long immunosuppression. The standard steroid therapy has strong side-effects and many patients are nonresponders to the therapy. Therefore, the goal for the treatment of AIH is specific immune regulation while preserving general immunocompetence. The use of antigen-specific regulatory $\mathrm{T}$ cells (Tregs) seems to be the most promising option. While the low frequency of natural antigen-specific Tregs and the resulting decreased possibility of their identification creates large problems, clinically used $\mathrm{T}$ cells will need genetically modified $\mathrm{T}$ cell receptors. Unfortunately, $\mathrm{T}$ cells with a classical transgenic $\mathrm{T}$ cell receptor are not of high clinical value due to MHC restriction. Therefore, the use of chimeric antigen receptors (CARs) seems to be a favorable option.
\end{abstract}

Abbreviations: AIH: Autoimmune Hepatitis; CARs: Chimeric Antigen Receptors; MS: Multiple Sclerosis, T1D: Type 1 Diabetes, HBV: Hepatitis B Virus, HCV: Hepatitis C Virus; HEV: Hepatitis E Virus; EAE: Experimental Autoimmune Encephalomyelitis; scFv: Single Chain Fragment ()

\section{The Liver as an Immune-Privileged Area and the Consequences}

The immunological functions and the tolerogenic properties of the liver are unique. We have known for decades that transplanted livers are better tolerated and rejected less often than other transplanted organs [1]. Autoimmune diseases of the liver, such as autoimmune hepatitis (AIH) [2], primary biliary cirrhosis [3] and primary sclerosing cholangitis, have a relatively low frequency compared to other autoimmune diseases, such as type 1 diabetes (T1D), multiple sclerosis (MS), rheumatoid arthritis and thyroiditis.

This high level of tolerance is immensely important. Among other things, many antigens from the gut of healthy individuals reach the immune system of the liver through the gut-liver axis. This occurs even more often under the conditions of intestinal disease with degradation of the mucosal barrier. The liver is one of the first organs that contacts neoantigens from the gut. The evolutionary price of this privilege and the high regenerative capacity of the liver is its susceptibility to chronic viral infections such as hepatitis B virus (HBV), hepatitis $\mathrm{C}$ virus (HCV) and hepatitis E virus (HEV) without the immediate destruction of the target cells. In these situations, the immune privilege of the liver contributes to the lack of infection control by the host's adaptive immune system.

Although the liver is a tolerogenic environment, autoimmune diseases such as AIH still occur. AIH is a serious, chronic liver disease affecting both children and adults. The diagnosis of AIH is often years to decades after its induction. AIH occurs in genetically predisposed individuals when a trigger, such as exposure to a virus such as HEV [4,5] results in a T cell-mediated autoimmune response to liver autoantigens. This immune response is made possible by inadequate regulatory immune control or the described immune privilege, which then leads to a loss of tolerance.

AIH often responds favorably to immunosuppressive treatment. Standard regimens involve relatively high starting doses of corticosteroids, which must be taken at low levels 
throughout life. For patients who do not respond to standard treatment, mycophenolate mofetil or calcineurin inhibitors are used. In any case, the side effects of this therapy are severe. If therapy fails or AIH remains untreated, liver transplantation is the last life-saving option for those who reach end-stage liver disease [6]. However, AIH may even recur or develop after transplantation (Figure 1).

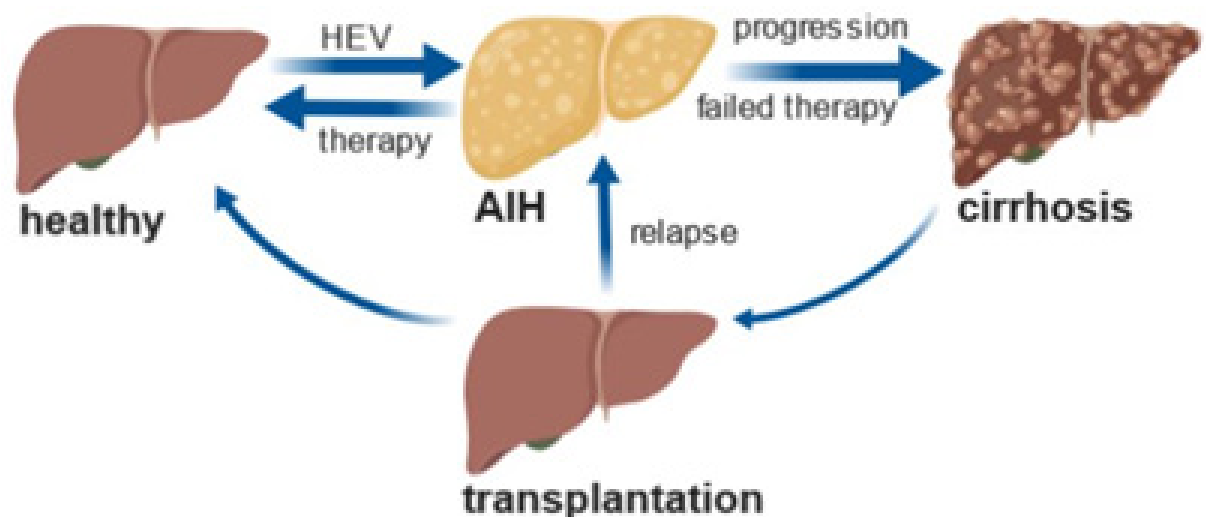

Figure 1: Induction of AIH by HEV, progression to cirrhosis and transplantation as last life-saving option (generated by https:// biorender.com).

\section{In vivo Tolerance Induction by Regulatory T Cells}

The goal for the treatment of AIH and all other autoimmune diseases is specific immune regulation while preserving general immunocompetence. One promising idea is the induction of antigen-specific regulatory T cells (Tregs) in the context of AIH. The general immunosuppression that many corticosteroid drugs achieve is thus avoided. Instead, antigen-specific tolerance to autoantigens is promoted.

Such approaches already exist to treat T1D and MS. Instead of transferring antigen-specific Tregs, these gene therapies either induce insulin expression in T1D or neuronal antigens in experimental autoimmune encephalomyelitis (EAE) in the tolerogenic liver microenvironment. The expression of these antigens within the liver prevents the development of EAE or T1D [7]. The induction of antigen-specific CD4+CD25+Foxp3+ Tregs was shown to protect against EAE $[8,9]$. The targeted gene transfer of autoantigens into hepatocytes appears to favor the systemic induction of antigen-specific Tregs. However, this form of autoimmune liver disease treatment will reach its limit, as tolerance would be induced for antigens that are expressed in the liver anyway. The utilization of the gut-liver axis and the introduction of autoantigens to take advantage of oral tolerance induction would involve less risk than the genetic transfer of autoantigens into hepatocytes. We and others showed that Foxp3+ Tregs can arise in this way [10]. The induction of hepatic antigens has not yet been demonstrated; however, the model antigen data are promising $[11,12]$. Another possibility is the additional administration of IL-2 to enrich existing Tregs.

The ThRIL study (NCT02166177) has already showed the positive influence of IL-2 after the transfer of adoptive Treg cells after liver transplantation [13]. This is remarkable because a decrease in the number of Tregs does not seem to be the problem, even during acute rejection after transplantation [14]. This is a comparable situation to patients with active AIH. Tregs and effector
T cells (Teffs) are enriched in the liver with a constant Treg / Teff ratio $[15,16]$. More importantly, immunosuppression with steroids and azathioprine resulted in the disproportionate loss of intrahepatic Tregs. Patients who achieved a biochemical response had higher intrahepatic Treg to Teff cell ratios than patients who did not achieve remission. The proliferation of Tregs was more suppressed by prednisolone than the expansion of Teffs. Against this background, it is noteworthy that one out of two AIH patients who did not respond to azathioprine showed a marked improvement in AIH after low-dose IL-2 therapy [17].

\section{Perspective: Adoptive Cell Therapy with Tregs}

Studies are underway to assess the safety and feasibility of autologous, polyclonal, or alloantigen-specific Treg transfer in organ transplantation, i.e., the aforementioned ThRIL, TRACT (NCT02145325) and TASK (NCT02711826) studies. Antigenspecific Tregs are superior to polyclonal Tregs in their suppression $[18,19]$. As opposed to nonspecific immunosuppression mediated by polyclonal Tregs, CAR-Treg-mediated inhibition is antigen-specific. This may cause fewer side effects associated with immunosuppression by polyspecific Tregs. While antigenspecific Tregs can be identified relatively well in the context of transplantation or graft-versus-host disease, since approximately 10 percent of all $\mathrm{T}$ cells (including Tregs) are allospecific [19], which places the problem of autoimmunity in a different light. The very low frequency of Tregs and the resulting decreased possibility of their identification creates large problems. Therefore, their subsequent purification according to GMP standards and expansion under GMP conditions threatens to become very difficult on a clinical scale according to today's standards and technology.

$\mathrm{T}$ cells with a transgenic $\mathrm{T}$ cell receptor are often potent but not of high clinical value due to MHC restriction, as the system would need to be individually tailored to each patient and therefore would not be transferable in the clinic.A new silver lining on the horizon is the use of chimeric antigen receptors (CARs). In contrast to the 
T cell receptors, CARs consist of a single polypeptide chain with an extracellular antibody-derived binding domain or a single chain fragment (scFv), the intracellular CD3-zeta signal domain and often a costimulatory domain such as CD28. These CARs showed great success in effector $\mathrm{T}$ cells against $\mathrm{B}$ cell lymphoma and received FDA approval. However, the CARs can also modify the specificity of Tregs. This was demonstrated in early preclinical studies on transplant rejection [20-22], EAE [23], colitis [24] and asthma [25].

However, there are some serious limitations to CAR-Tregs. It is well known that anti-tumor CAR-T cell treatments causes side effects including cytokine storm [26] but also neurotoxicity [27]. Although it is unlikely due to the function of Tregs, it remains to be seen in early studies whether CAR-Tregs trigger these side effects as well. Also important is the choice of antigen, which recognizes the ScFv and thus defines the antigen specificity of the CARs. The antigen itself and the affinity between the antigen and the scFv appear to have a significant impact on the functionality of the CAR. The costimulatory domain on the CAR, making it a secondgeneration CAR, is essential to prevent $\mathrm{T}$ cells from becoming anergic. The approach to the use of CAR-Tregs involves second generation CARs with a CD28 costimulatory domain. However, studies have shown that CARs with a CD137 costimulatory domain prevented $\mathrm{T}$ cell exhaustion and thus improved the durability of the CAR-T cells. This was not observed with CD28 costimulation $[28,29]$. There is much space for improvement, and the use of other costimulatory domains such as OX40L may further help to maintain CAR-Tregs.

The insertion of CARs into $\mathrm{T}$ cells and Tregs also brings up critical questions. Most approaches prefer retro- or lentiviral transfer, in which the endogenous $\mathrm{T}$ cell receptor remains intact. If this is replaced by CRISPR-Cas 9 targeting, the question remains of how targeted this technology is in practice [30,31]. It remains to be seen which of these technologies will ultimately be used as an alternate after failed steroid therapy or even as a replacement of steroids as new first-line therapy.

\section{References}

1 Calne RY, Sells RA, Pena JR, Davis DR, Millard PR, et al. (1969) Induction of immunological tolerance by porcine liver allografts. Nature 223(5205): 472-476.

2 Alvarez F, Berg PA, Bianchi FB, Bianchi L, Burroughs AK, et al. (1999) International autoimmune hepatitis group report: Review of criteria for diagnosis of autoimmune hepatitis. J Hepatol 31(5): 929-938.

3 Ichiki Y, Shimoda S, Ishibashi H, Gershwin ME (2004) Is primary biliary cirrhosis a model autoimmune disease? Autoimmun Rev 3(4): 331-336.

4 Pischke S, Gisa A, Suneetha PV, Wiegand SB, Taubert R, et al. (2014) Increased HEV seroprevalence in patients with autoimmune hepatitis. PLoS One 9(1): e85330.

5 Taubert R, Diestelhorst J, Junge N, Kirstein MM, Pischke S, et al. (2018) Increased seroprevalence of HAV and parvovirus B19 in children and of HEV in adults at diagnosis of autoimmune hepatitis. Sci Rep 8(17452).

6 Czaja AJ (2016) Diagnosis and management of autoimmune hepatitis: Current status and future directions. Gut Liver 10(2): 177-203.

7 Akbarpour M, Goudy KS, Cantore A, Russo F, Sanvito F, et al. (2015) Insulin $B$ chain 9-23 gene transfer to hepatocytes protects from type 1 diabetes by inducing Ag-specific FoxP3+ Tregs. Sci Transl Med 7(289): 289 ra81.

8 Luth S, Huber S, Schramm C, Buch T, Zander S, et al. (2008) Ectopic expression of neural autoantigen in mouse liver suppresses experimental autoimmune neuroinflammation by inducing antigen-specific tregs. the journal of clinical investigation 118(10): 3403-3410.

9 Keeler GD, Kumar S, Palaschak B, Silverberg EL, Markusic DM, et al. (2018) Gene therapy-induced antigen-specific tregs inhibit neuroinflammation and reverse disease in a mouse model of multiple sclerosis. Mol Ther 26(1): 173-183.

10 Hadis U, Wahl B, Schulz O, Hardtke-Wolenski M, Schippers A, et al. (2011) Intestinal tolerance requires gut homing and expansion of Foxp3(+) regulatory t cells in the lamina propria. Immunity 34(2): 237-246.

11 Hauet-Broere F, Unger WW, Garssen J, Hoijer MA, Kraal G, et al. (2003) Functional CD25- and CD25+ mucosal regulatory T cells are induced in gut-draining lymphoid tissue within $48 \mathrm{hr}$ after oral antigen application. Eur J Immunol 33(10): 2801-2810.

12 Wang X, Sherman A, Liao G, Leong KW, Daniell H, et al. (2013) Mechanism of oral tolerance induction to therapeutic proteins. Adv Drug Deliv Rev 65(6): 759-773.

13 Safinia N, Vaikunthanathan T, Fraser H, Thirkell S, Lowe K, et al. (2016) Successful expansion of functional and stable regulatory $\mathrm{T}$ cells for immunotherapy in liver transplantation. Oncotarget 7(7): 7563-7577.

14 Taubert R, Pischke S, Schlue J, Wedemeyer H, Noyan F, et al. (2012) Enrichment of regulatory $\mathrm{T}$ cells in acutely rejected human liver allografts. Am J Transplant 12(12): 3425-3436.

15 Taubert R, Hardtke-Wolenski M, Noyan F, Wilms A, Baumann AK, et al. (2014) Intrahepatic regulatory $T$ cells in autoimmune hepatitis are associated with treatment response and depleted with current therapies. J Hepatol 61(5): 1106-1114.

16 Peiseler M, Sebode M, Franke B, Wortmann F, Schwinge D, Quaas A (2012) FOXP3+ regulatory T cells in autoimmune hepatitis are fully functional and not reduced in frequency. J Hepatol 57(1):125-132

17 Lim TY, Martinez-Llordella M, Kodela E, Gray E, Heneghan MA (2018) Low-dose interleukin-2 for refractory autoimmune hepatitis. Hepatology 68(4): 1649-1652.

18 Noyan F, Lee YS, Hardtke-Wolenski M, Knoefel AK, Taubert R, et al. (2013) Donor-specific regulatory T cells generated on donor B cells are superior to $\mathrm{CD} 4+\mathrm{CD} 25 \mathrm{high}$ cells in controlling alloimmune responses in humanized mice. Transplant Proc 45(5): 1832-1837.

19 Noyan F, Lee YS, Zimmermann K, Hardtke-Wolenski M, Taubert R, et al. (2014) Isolation of human antigen-specific regulatory T cells with high suppressive function. Eur J Immunol 44(9): 2592-2602.

20 Noyan F, Zimmermann K, Hardtke-Wolenski M, Knoefel A, Schulde E (2017) Prevention of allograft rejection by use of regulatory t cells with an MHC-Specific chimeric antigen receptor. Am J Transplant 17(4): 917930.

21 Boardman DA, Philippeos C, Fruhwirth GO, Ibrahim MA, Hannen RF (2017) Expression of a chimeric antigen receptor specific for donor HLA class I enhances the potency of human regulatory $t$ cells in preventing human skin transplant rejection. Am J Transplant 17(4): 931-943.

22 MacDonald KG, Hoeppli RE, Huang Q Gillies J, Luciani DS, et al. (2016) Alloantigen-specific regulatory $\mathrm{T}$ cells generated with a chimeric antigen receptor. J Clin Invest 126(4): 1413-1424

23 Fransson M, Piras E, Burman J, Nilsson B, Essand M (2012) CAR/FoxP3engineered $\mathrm{T}$ regulatory cells target the CNS and suppress EAE upon intranasal delivery. J Neuroinflammation 9: 112.

24 Blat D, Zigmond E, Alteber Z, Waks T, Eshhar Z (2014) Suppression of murine colitis and its associated cancer by carcinoembryonic antigenspecific regulatory T cells. Mol Ther 22(5): 1018-1028. 
25 Skuljec J, Chmielewski M, Happle C, Habener A, Busse M (2017) Chimeric antigen receptor-redirected regulatory $\mathrm{T}$ cells suppress experimental allergic airway inflammation, a model of asthma. Front Immunol 8: 1125 .

26 Morgan RA, Yang JC, Kitano M, Dudley ME, Laurencot CM, et al. (2010) Case report of a serious adverse event following the administration of $\mathrm{T}$ cells transduced with a chimeric antigen receptor recognizing ERBB2. Mol Ther 18(4): 843-851.

27 Hunter BD, Jacobson CA (2019) CAR T-cell associated neurotoxicity: Mechanisms, clinicopathologic correlates, and future directions. J Natl Cancer Inst.
28 Long AH, Haso WM, Shern JF, Wanhainen KM, Murgai M, et al. (2015) 4-1BB stimulation ameliorates $\mathrm{T}$ cell exhaustion induced by tonic signalling of chimeric antigen receptors. Nat Med 21(6): 581-590.

29 Quintarelli C, Orlando D, Boffa I, Guercio M, Polito VA, et al. (2018) Choice of costimulatory domains and of cytokines determines CAR T-cell activity in neuroblastoma. Oncoimmunology 7(6): e1433518.

30 Zhang XH, Tee LY, Wang XG, Huang QS, Yang SH (2015) Off-target effects in CRISPR/Cas9-mediated Genome Engineering. Mol Ther Nucleic Acids 4: e264.

31 Peng R, Lin G, Li J (2016) Potential pitfalls of CRISPR/Cas9-mediated genome editing. FEBS J 283(7): 1218-1231. 